\title{
Inactivation of RUNX3 protein expression in tongue squamous cell carcinoma and its association with clinicopathological characteristics
}

\author{
WEI-NA ZHOU ${ }^{1,2^{*}}$, YI-FEI DU ${ }^{1,3 *}$, YANG ZHENG $^{1,3}$, WEI ZHANG $^{1,4}$, \\ YU-NONG WU ${ }^{1,3}$, XIAO-MENG SONG ${ }^{1,3}$ and JIN BAI \\ ${ }^{1}$ Jiangsu Key Laboratory of Oral Diseases; Departments of ${ }^{2}$ Polyclinic Dentistry, \\ ${ }^{3}$ Oral and Maxillofacial Surgery and ${ }^{4}$ Pathology, Affiliated Hospital of Stomatology, Nanjing Medical University, \\ Nanjing, Jiangsu 210029; ${ }^{5}$ Jiangsu Center for The Collaboration and Innovation of Cancer Biotherapy, \\ Cancer Institute, Xuzhou Medical University, Xuzhou, Jiangsu 221002, P.R. China
}

Received January 26, 2018; Accepted October 17, 2018

DOI: $10.3892 / \mathrm{mmr} .2018 .9705$

\begin{abstract}
The function of runt-related transcription factor 3 (RUNX3) in oral cancer remains controversial. The present study aimed to determine the status of RUNX3 protein expression and its association with clinicopathological characteristics in tongue squamous cell carcinomas (SCC). The present study used three pairs of tongue SCC and non-cancerous tissues to assess RUNX3 protein expression by western blot analysis, and two tongue SCC cell lines to determine RUNX3 protein localization by immunofluorescence and immunocytochemistry. Tissue microarray immunohistochemistry was performed to detect the clinical relevance of RUNX3 in 79 patients with tongue SCC. The results demonstrated that RUNX3 protein expression was reduced in tongue SCC tissues compared with in paired non-cancerous tissues. Similarly, the expression of RUNX3 was low in SCC25 and Cal27 cells, and was predominantly localized to the cytoplasm. In the 79 patients with tongue SCC, RUNX3 protein expression was presented in different manners in carcinoma nests and tumor stroma. RUNX3 in carcinoma nests (nRUNX3) exhibited nuclear positive staining in 24/79 samples, cytoplasmic mislocalization in 41/79 samples and was undetectable in 14/79 samples. RUNX3
\end{abstract}

Correspondence to: Dr Xiao-Meng Song, Jiangsu Key Laboratory of Oral Diseases, Affiliated Hospital of Stomatology, Nanjing Medical University, 140 Hanzhong Road, Nanjing, Jiangsu 210029, P.R. China E-mail: sxm2081@163.com

Professor Jin Bai, Jiangsu Center for The Collaboration and Innovation of Cancer Biotherapy, Cancer Institute, Xuzhou Medical University, 84 West Huaihai Road, Xuzhou, Jiangsu 221002, P.R. China

E-mail: wanlinzhong2006@163.com

*Contributed equally

Key words: runt-related transcription factor 3, tongue squamous cell carcinoma, cytoplasmic mislocalization, prognosis in stroma (sRUNX3) exhibited nuclear positive staining in 40/79 samples and nuclear negative staining in 39/79 samples. Negative nRUNX3 expression was significantly associated with lymph node metastasis $(\mathrm{P}=0.014)$, clinical stage $(\mathrm{P}=0.027)$, and overall and disease-free survival $(\mathrm{P}=0.008$ and $\mathrm{P}=0.007$, respectively). In addition, negative sRUNX3 expression was associated with lymph node metastasis $(\mathrm{P}=0.003)$ and clinical stage $(\mathrm{P}=0.003)$; however, not with overall survival. The findings of the present study preliminarily suggested that cytoplasmic mislocalization of RUNX3 protein may be a common event in tongue SCC, and that sRUNX3 protein expression may be a potential prognostic biomarker.

\section{Introduction}

Annually, >275,000 novel cases of oral cancer worldwide are reported and two-thirds of these cases occur in developing regions, including Southeast Asia (1). Tongue squamous cell carcinoma (SCC) is the most common subtype of oral cancer, which accounts for $40-50 \%$ of all cases (2). In recent years, the incidence rate of tongue SCC has significantly increased, particularly among young adults (3). Furthermore, a number of patients with tongue SCC present with local lymph node metastasis at the time of diagnosis. Despite the development of comprehensive therapies, including extensive resection, and chemo- and radiotherapy committed to tongue SCC, the prognosis remains poor due to tumor recurrence and metastasis. For prognostic prediction to improve the survival rate of patients with fewer therapy-associated complications, it has become urgent to seek potential biomarkers for tongue SCC (4).

The runt-related transcription factor (RUNX) family has three members: RUNX1, RUNX2 and RUNX3 (5). The RUNX family encodes proteins that contain a well-conserved 128 amino acid region (Runt domain), which form a stable complex with core-binding factor subunit $\beta$ to exert their transactivation ability. RUNX proteins may regulate target genes by binding to the promoters or enhancers of these genes, which become disrupted in cancer cells (6). Among these three RUNX family members, RUNX3 has been identified to be 
closely associated with tumorigenesis and/or tumor progression (7). Absence of RUNX3 expression has been detected in various types of human cancer, including esophageal, gastric, colorectal, lung and breast cancer (8-12). Inactivation of RUNX3 is additionally significantly correlated with lymph node metastasis and worse prognosis. Conversely, the restoration of RUNX3 may suppress the migration and invasion of cancer cells in vitro (13-16). These previous studies suggested that RUNX3 exerts tumor suppressive effects and may serve a critical role in the development of tumor metastasis. However, the function of RUNX3 in tongue SCC has yet to be fully elucidated.

The present study aimed to detect RUNX3 expression in patients with tongue SCC using tissue microarray (TMA) technology and to analyze its association with clinicopathological parameters. In addition, the present study aimed to analyze the characteristics of RUNX3 in clinical samples and tongue SCC cell lines.

\section{Patients and methods}

Ethics statement. The present study was performed under a protocol approved by the Institutional Review Boards of the Affiliated Hospital of Stomatology, Nanjing Medical University (Nanjing, China). All patients provided written informed consent prior to the study.

Patient samples. In total, three cancerous and paired non-cancerous tissues were obtained from patients with tongue SCC without a history of smoking or alcohol consumption. All three patients were admitted to the Department of Oral and Maxillofacial Surgery, Stomatological Hospital Affiliated to Nanjing Medical University in 2015. The patients comprised two men: i) Age, 46 years; admitted in December, 2015; ii) age, 56 years; admitted in May, 2015, and one woman (age, 68 years; admitted in December, 2015). All patients were pathologically diagnosed with moderate SCC and T1N0M0. The fresh samples were washed three times with sterilized PBS and lysed in ice-cold lysis buffer (Becton, Dickinson and Company, Franklin Lakes, NJ, USA) for $2 \mathrm{~h}$ at $4^{\circ} \mathrm{C}$. The samples were subsequently centrifuged at $12,000 \mathrm{x} \mathrm{g}$ for $30 \mathrm{~min}$ at $4^{\circ} \mathrm{C}$, the supernatants were collected and total protein concentration was measured by a bicinchoninic acid protein assay (Invitrogen; Thermo Fisher Scientific, Inc., Waltham, MA, USA) for RUNX3 protein detection.

Western blot analysis. Tissue proteins $(10 \mu \mathrm{g})$ were separated by $12 \%$ SDS-PAGE, blocked with $5 \%$ bovine serum albumin (cat. no. 36101ES25; Yeasen Biological Technology, Ltd., Shanghai, China) room temperature for $2 \mathrm{~h}$, and subsequently transferred onto a nitrocellulose membrane. The member was incubated overnight at $4^{\circ} \mathrm{C}$ with monoclonal anti-RUNX3 (cat. no. ab135248; 1:2,000; Abcam, Cambridge, UK) and mouse anti- $\beta$-actin $(1: 1,000$; cat. no. BA5180; Wuhan Boster Biological Technology, Ltd., Wuhan, China). Membranes were washed with PBS, incubated with secondary antibodies [goat anti-rabbit (1:3,000; cat. no. BA1056; Wuhan Boster Biological Technology, Ltd.) and goat anti-mouse immunoglobulin G (IgG; 1:3,000; cat. no. BA1050; Wuhan Boster Biological Technology, Ltd.)] for $2 \mathrm{~h}$ at room temperature and washed with PBS containing 0.05\% Tween-20 at room temperature. Subsequently, visualization fluid (containing $10 \mathrm{ml}$ alkaline phosphatase buffer, $33 \mu 1$ 5-bromo-4-chloro-3-indolyl phosphate and $66 \mu 1$ nitro-blue tetrazolium chloride) was added to the membrane and the protein bands were detected using Super Signal West Pico Chemiluminescent Substrate (Thermo Fisher Scientific, Inc.). In total, three independent blots of each protein were semi-quantified using Image $\mathrm{J}$ software (1.48 version; National Institutes of Health, Bethesda, MD, USA).

Tongue SCC cell culture. Human tongue SCC cell lines SCC25 and Cal27 were purchased from the Shanghai Institute of Biochemistry and Cell Biology, Chinese Academy of Sciences (Shanghai, China). SCC25 and Cal27 cells were cultured in RPMI 1640 medium supplemented with $10 \%$ fetal calf serum (both Gibco; Thermo Fisher Scientific, Inc.) at $37^{\circ} \mathrm{C}$ in a humidified incubator containing $95 \%$ air and $5 \% \mathrm{CO}_{2}$.

Immunofluorescence. SCC25 and Cal27 cells $\left(0.5 \times 10^{5}\right)$ were seeded on coverslips and cultured for $24 \mathrm{~h}$ at $37^{\circ} \mathrm{C}$; subsequently, the coverslips were washed with PBS, fixed with $4 \%$ paraformaldehyde for $30 \mathrm{~min}$ at room temperature, permeabilized in PBS containing $0.25 \%$ Triton X-100 for $30 \mathrm{~min}$ at room temperature and incubated with $10 \%$ normal rabbit serum (cat. no. BSD0009; Yuduo Bio., Shanghai, China) for $30 \mathrm{~min}$ at room temperature, in order to block nonspecific staining. Cells were then incubated overnight at $4{ }^{\circ} \mathrm{C}$ with mouse anti-RUNX3 antibody (1:500) and were incubated with fluorescein isothiocyanate-conjugated secondary IgG (1:50; cat. no. SA1064; Wuhan Boster Biological Technology, Ltd. Wuhan, China) for $2 \mathrm{~h}$ at room temperature. Cells were stained with DAPI (1:1,000; cat. no. c1002; Beyotime Institute of Biotechnology, Haimen, China) for $1 \mathrm{~min}$ to label the nuclei and were subsequently washed with PBS. PBS was used instead of a primary antibody as a negative control. Fluorescence images were captured under a fluorescence microscope (Leica Microsystems GmbH, Wetzlar, Germany; magnification, x200).

Immunocytochemistry. SCC 25 and Cal27 cells ( $0.5 \times 10^{5}$ cells) were seeded on coverslips and were processed using the same protocol as for immunofluorescence. Following an overnight incubation at $4^{\circ} \mathrm{C}$ with the RUNX3 (1:500) antibody, cells were subjected to the streptavidin-peroxidase (Sp) method using a standard Sp kit (cat. no. PV-9000; Zhongshan Bio-Tech Co., Ltd., Beijing, China) according to the manufacturer's protocol. Immunoreactivity was assessed and representative images were captured using a light microscope with AxioCam MRc5 camera (Carl Zeiss AG, Oberkochen, Germany; magnification, x200).

TMA immunohistochemistry. Between January 2009 and January 2014, tissues were collected during surgery from 79 patients with tongue SCC hospitalized in the Department of Oral and Maxillofacial Surgery, Affiliated Hospital of Stomatology, Nanjing Medical University, in order to manufacture a tongue SCC TMA. Patients who had received preoperative radiotherapy or chemotherapy were excluded from the study and two pathologists confirmed all cases. All patients were staged according to the classification of oral cancer by the National Comprehensive Cancer Network (17) 
A

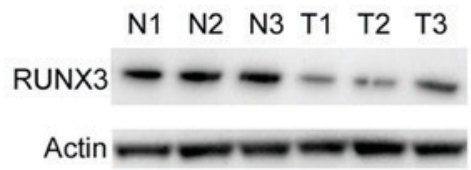

C
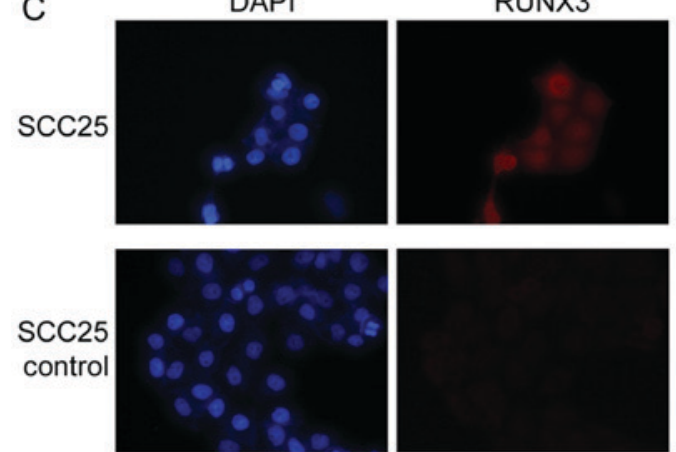

B

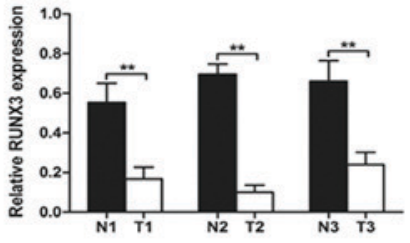

Merged
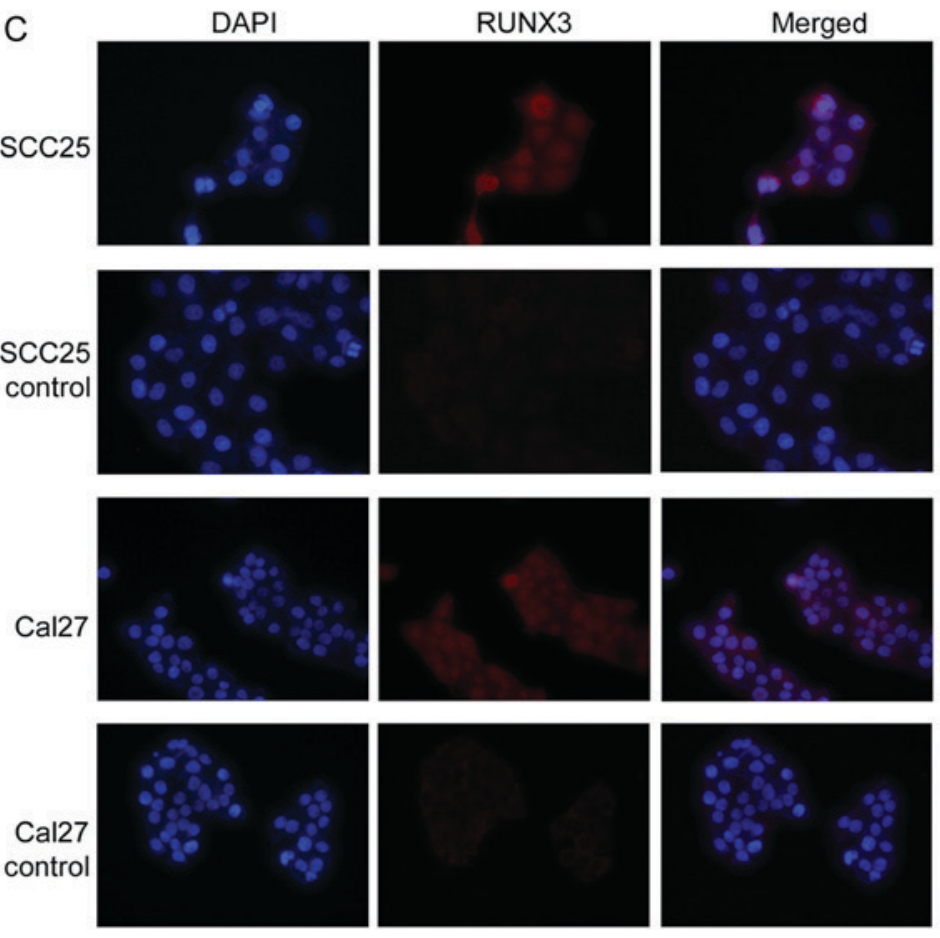

D
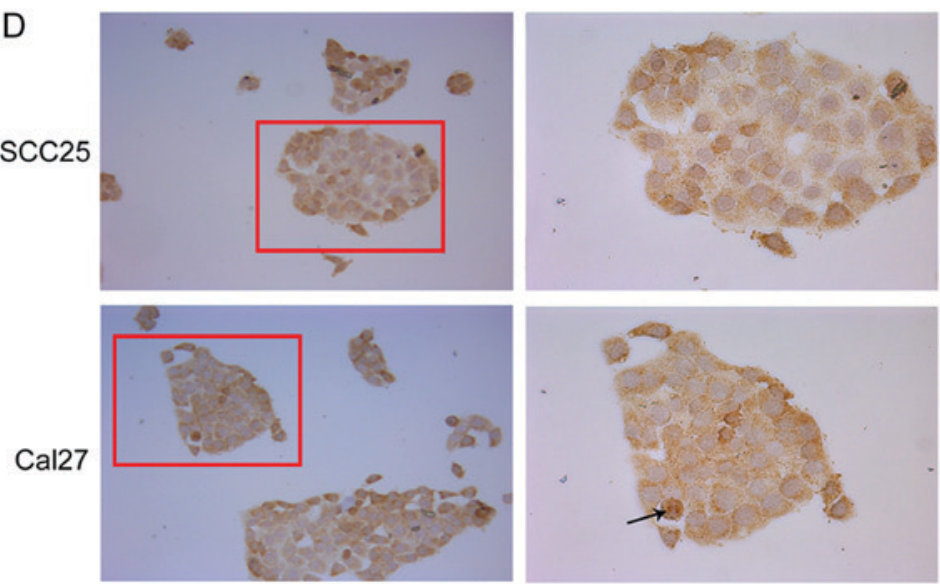

Figure 1. Expression and localization of RUNX3 in tongue SCC. (A and B) Western blot analysis of the relative protein expression levels of RUNX3 in three tongue SCC tissues and paired non-cancerous tissues. ${ }^{* *} \mathrm{P}<0.01$. (C) Immunofluorescence of RUNX3 in tongue SCC cell lines SCC25 and Cal27; low expression was detected in the cytoplasm (magnification, x200). Control groups were incubated with PBS instead of first antibody. (D) Immunochemistry of RUNX3 in tongue SCC cell lines SCC25 and Cal27 (left panel, magnification, x100; right panel, magnification, x200). The majority of staining was detected in the cytoplasm, but a number of individual cells presented with nuclear staining (black arrow). N, non-cancerous tissue; RUNX3, runt-related transcription factor 3; SCC, squamous cell carcinoma; T, tumor tissue.

(2015) and were followed up at least 24 months post-operation for prognostic analysis. The array dot diameter was $1.5 \mathrm{~mm}$, and each dot represented a tissue spot from one individual specimen that had been selected and pathologically confirmed (18).

Immunohistochemistry was performed according to the Sp method using a standard Sp kit (OriGene Technologies, Inc.). The tongue SCC TMA slide was incubated with the monoclonal RUNX3 antibody (1:250) overnight at $4^{\circ} \mathrm{C}$. Cells were subjected to the Sp method (cat. no. PV-9000; Zhongshan
Bio-Tech Co., Ltd.) according to the manufacturer's protocol Diaminobenzidine (OriGene Technologies, Inc. Beijing, China) was used as a chromogen for the immunoperoxidase reaction. The slides were counterstained with hematoxylin for $5 \mathrm{~min}$ at room temperature. Non-immune serum (cat. no. BSD0009; Yuduo Bio.) instead of first antibody was used as a negative control.

Immunoreactivity in carcinoma nests and stroma was blindly assessed by two independent observers using a Zeiss 
Table I. nRUNX3 and its clinicopathological characteristics in 79 patients with tongue squamous cell carcinoma.

\begin{tabular}{|c|c|c|c|c|}
\hline \multirow[b]{2}{*}{ Variable } & \multicolumn{2}{|c|}{ nRUNX3 staining } & \multirow[b]{2}{*}{ Total } & \multirow[b]{2}{*}{ P-value ${ }^{a}$} \\
\hline & Negative (\%) & Positive (\%) & & \\
\hline \multicolumn{5}{|l|}{ Age, years } \\
\hline$\geq 60$ & $23(74.2)$ & $8(25.8)$ & 31 & \multirow[t]{2}{*}{0.618} \\
\hline$<60$ & $32(66.7)$ & $16(33.3)$ & 48 & \\
\hline \multicolumn{5}{|l|}{ Sex } \\
\hline Male & $30(69.8)$ & $13(30.2)$ & 43 & \multirow[t]{2}{*}{0.975} \\
\hline Female & $25(69.4)$ & 11 (30.6) & 36 & \\
\hline \multicolumn{5}{|l|}{ Tumor size, cm } \\
\hline$\leq 2$ & $25(71.0)$ & $16(29.0)$ & 41 & \multirow[t]{2}{*}{0.093} \\
\hline$>2$ & $30(78.9)$ & $8(21.1)$ & 38 & \\
\hline \multicolumn{5}{|c|}{ Lymph node status } \\
\hline No & $21(55.3)$ & $17(44.7)$ & 38 & \multirow[t]{2}{*}{0.014} \\
\hline $\mathrm{N} 1,2$ and 3 & $34(82.9)$ & 7 (17.1) & 41 & \\
\hline \multicolumn{5}{|c|}{ Histological grade } \\
\hline I & $30(68.2)$ & $14(31.8)$ & 44 & \multirow[t]{2}{*}{0.809} \\
\hline II and III & 25 (71.4) & $10(28.6)$ & 35 & \\
\hline \multicolumn{5}{|l|}{ Clinical stage } \\
\hline I & $23(57.5)$ & $17(42.5)$ & 40 & \multirow[t]{3}{*}{0.027} \\
\hline II, III, and IV & $32(82.1)$ & $7(17.9)$ & 39 & \\
\hline Total & 55 & 24 & 79 & \\
\hline
\end{tabular}

${ }^{a}$ Fisher's exact test. nRUNX3, RUNX3 in carcinoma nests; RUNX3, runt-related transcription factor 3.

Imager Z1 light microscope (magnification, x100), and the representative images were obtained using an AxioCam MRc5 camera (both Carl Zeiss AG). Carcinoma nests were defined as a cluster of cancer cells and the connective tissues around the nests were considered as tumor stroma. RUNX3 immunoreactivity in nuclei or cytoplasm was graded as follows: Positive expression (positive cells $\geq 5 \%$ ) and negative expression (positive cells $<5 \%$ ) (19).

Statistical analysis. Statistical analysis was performed using SPSS software version 20 (IBM Corp., Armonk, NY, USA). The western blotting data were analyzed using a paired Student's t-test. The associations between RUNX3 staining and clinicopathological parameters were evaluated by Fisher's exact test and $\chi^{2}$ test. The association between RUNX3 expression in carcinoma nests/stroma and patient survival was analyzed by Kaplan-Meier survival analysis and a log-rank test. Univariate and multivariate Cox regression analyses were used to investigate whether RUNX3 expression in carcinoma nests may be a predictor of overall survival in patients with tongue SCC. $\mathrm{P}<0.05$ was considered to indicate a statistically significant difference.

\section{Results}

Reduced expression of RUNX3 protein in tongue SCC. RUNX3 expression was significantly reduced in tongue SCC tissues compared with paired non-cancerous tissues, indicating that RUNX3 may be inactivated in patients with tongue SCC
(Fig. 1A and $\mathrm{B} ; \mathrm{P}<0.01$ ). To confirm this observation, immunofluorescence and immunocytochemistry were used to detect RUNX3 expression in the human tongue SCC cell lines, SCC25 and Cal27. Immunofluorescence demonstrated that there was low RUNX3 expression in the cytoplasm (Fig. 1C), thus confirming that RUNX3 was localized to the cytoplasm in SCC25 and Cal27 cells. Immunocytochemical analysis demonstrated similar results (Fig. 1D); however, nuclear staining of RUNX3 was detected in a number of tongue SCC cells (Fig. 1D).

Distinct expression of RUNX3 in tongue SCC nests and stroma. Immunohistochemistry of 79 patients with tongue SCC was performed using TMA technology to detect RUNX3 expression. The distribution of RUNX3-positive cells revealed that RUNX3 was expressed in different manners in carcinoma nests and stroma; to the best of our knowledge, this has not been reported in previous studies. RUNX3 in carcinoma nests (nRUNX3) exhibited nuclear staining in 24 cases $(30.4 \%)$. Cytoplasmic and undetectable staining were considered negative expression of RUNX3, with a total of 55 cases (Table I). RUNX3-positive staining in the nucleus and cytoplasm was not observed in any carcinoma nest. RUNX3 in stroma (sRUNX3) was nuclear positive in 40 cases $(50.6 \%)$ and nuclear negative in 39 cases (49.4\%; Table II).

As presented in Fig. 2A and B, positive nRUNX3 immunoreactivity was detected in the nuclei and cytoplasm of cancer cells. Cytoplasmic staining was distributed in the keratin pearl, and was the most common type of staining among 
Table II. sRUNX3 and its clinicopathological characteristics in 79 patients with tongue squamous cell carcinoma.

\begin{tabular}{|c|c|c|c|c|}
\hline \multirow[b]{2}{*}{ Variable } & \multicolumn{2}{|c|}{ sRUNX3 staining } & \multirow[b]{2}{*}{ Total } & \multirow[b]{2}{*}{ P-value } \\
\hline & Negative $(\%)$ & Positive (\%) & & \\
\hline \multicolumn{5}{|l|}{ Age, years } \\
\hline$\geq 60$ & $14(45.2)$ & $17(54.8)$ & 31 & 0.647 \\
\hline$<60$ & $25(52.1)$ & 23 (47.9) & 48 & \\
\hline \multicolumn{5}{|l|}{ Sex } \\
\hline Male & $24(55.8)$ & $19(44.2)$ & 43 & 0.261 \\
\hline Female & $15(41.7)$ & $21(58.3)$ & 36 & \\
\hline \multicolumn{5}{|l|}{ Tumor size, cm } \\
\hline$\leq 2$ & $19(46.3)$ & $22(53.7)$ & 41 & 0.655 \\
\hline$>2$ & $20(52.6)$ & $18(47.4)$ & 38 & \\
\hline \multicolumn{5}{|c|}{ Lymph node status } \\
\hline N0 & $12(31.6)$ & $26(68.4)$ & 38 & 0.003 \\
\hline $\mathrm{N} 1,2$ and 3 & $27(65.9)$ & $14(34.1)$ & 41 & \\
\hline \multicolumn{5}{|c|}{ Histological grade } \\
\hline I & $18(40.9)$ & $26(59.1)$ & 44 & 0.069 \\
\hline II and III & $21(60.0)$ & $14(40.0)$ & 35 & \\
\hline \multicolumn{5}{|l|}{ Clinical stage } \\
\hline $\mathrm{I}$ & $13(32.5)$ & $27(67.5)$ & 40 & 0.003 \\
\hline II, III, and IV & $26(66.7)$ & $13(33.3)$ & 39 & \\
\hline Total & 39 & 40 & 79 & \\
\hline
\end{tabular}

aFisher's exact test. RUNX3, runt-related transcription factor 3; sRUNX3, RNX3 in stroma.

the 79 cases. This 'RUNX3 mislocalization' suggested that RUNX3 protein was restricted to the cytoplasm and was not able to function as a tumor suppressor as reported in previous studies $(9,12)$. The reduced expression and mislocalization of RUNX3 protein were aberrant, thus indicating RUNX3 gene impairment in cancer cells; therefore, these two types of aberration were analyzed together as negative expression (Table I). As presented in Fig. 2C, the majority of positive sRUNX3 cells were detected in clumps in the connecting zone surrounding the carcinoma tissue. In addition, negative nRUNX3 and sRUNX3 staining appeared in certain cases (Fig. 2D).

Association of $n R U N X 3$ and sRUNX3 with clinicopathological parameters. The association between RUNX3 expression and clinicopathological variables was further investigated. It was demonstrated that positive nRUNX3 expression was more frequent in N0 cases and in clinical stage I cases (Fig. 3A and B). Positive sRUNX3 expression was also higher in N0 cases and clinical stage cases (Fig. 3C and D). Through statistical analysis, it was demonstrated that nRUNX3 expression was significantly associated with lymph node status and clinical stage $(\mathrm{P}=0.014$ and $\mathrm{P}=0.027$, respectively; Table I). In addition, sRUNX3 expression was significantly associated with lymph node status and clinical stage $(\mathrm{P}=0.003$ and $\mathrm{P}=0.003$, respectively; Table II). No significant associations were observed between the expression of nRUNX3 and sRUNX3 and other clinicopathological variables, including patient age, sex and tumor size.
Negative expression of $n R U N X 3$ is associated with poor patient survival. To determine whether RUNX3 expression was associated with the survival of patients with tongue SCC a Kaplan-Meier survival analysis was conducted. The data revealed that nRUNX3 expression was associated with overall patient survival and disease-free patient survival $(\mathrm{P}=0.008$ and $\mathrm{P}=0.007$, respectively, log-rank test; Fig. $3 \mathrm{E}$ and $\mathrm{F}$ ); however, this was not the case for sRUNX3 (data not shown).

Univariate Cox proportional hazards regression model was used to estimate the crude hazard ratios (HRs) of nRUNX3 expression or each clinicopathological variable on patient survival. The log-rank test and univariate Cox regression analyses revealed that nRUNX3 expression was significantly associated with overall survival in patients with tongue SCC [HR 3.71; 95\% confidence interval (CI) 1.30-10.60; $\mathrm{P}=0.015$; Table III]. To further validate the prognostic value of nRUNX3, multivariate analysis was performed and the significant factors are summarized in Table IV. The Cox regression model indicated that nRUNX3 expression was an independent prognostic marker for overall survival in patients with tongue SCC (HR 3.15; 95\% CI 1.04-9.51; $\mathrm{P}=0.042$, Table IV).

\section{Discussion}

While the overall incidence of oral cancer has barely altered in the last decade, the number of novel cases of tongue SCC significantly increases each year, particularly among young adults $(3,20)$. Tongue SCC exhibits a more aggressive 
Table III. Univariate Cox regression analysis of nRUNX3 expression and clinicopathological variables predicting the survival of patients with tongue squamous cell carcinoma.

\begin{tabular}{llr}
\hline & \multicolumn{2}{c}{$(\mathrm{n}=79$ cases $)$} \\
\cline { 2 - 3 } Variable & HR $(95 \% \mathrm{CI})$ & P-value \\
\hline Age $(<60$ vs. $\geq 60$ years) & $1.55(0.77-3.11)$ & 0.222 \\
Sex (male vs. female) & $0.81(0.40-1.65)$ & 0.567 \\
Tumor size ( $\leq 2$ cm vs. $>2 \mathrm{~cm})$ & $1.56(0.78-3.12)$ & 0.210 \\
Lymph node metastasis (N0 vs. N1/N2/N3) & $1.62(0.79-3.31)$ & 0.190 \\
Histological grade (I vs. II/III) & $1.21(0.60-2.41)$ & 0.596 \\
Clinical stage (I vs. II/III/IV) & $1.11(0.56-2.24)$ & 0.762 \\
nRUNX3 expression (negative vs. positive) & $3.71(1.30-10.60)$ & 0.015 \\
\hline
\end{tabular}

CI, confidence interval; HR, hazard ratio; nRUNX3, runt-related transcription factor 3 in carcinoma nests.
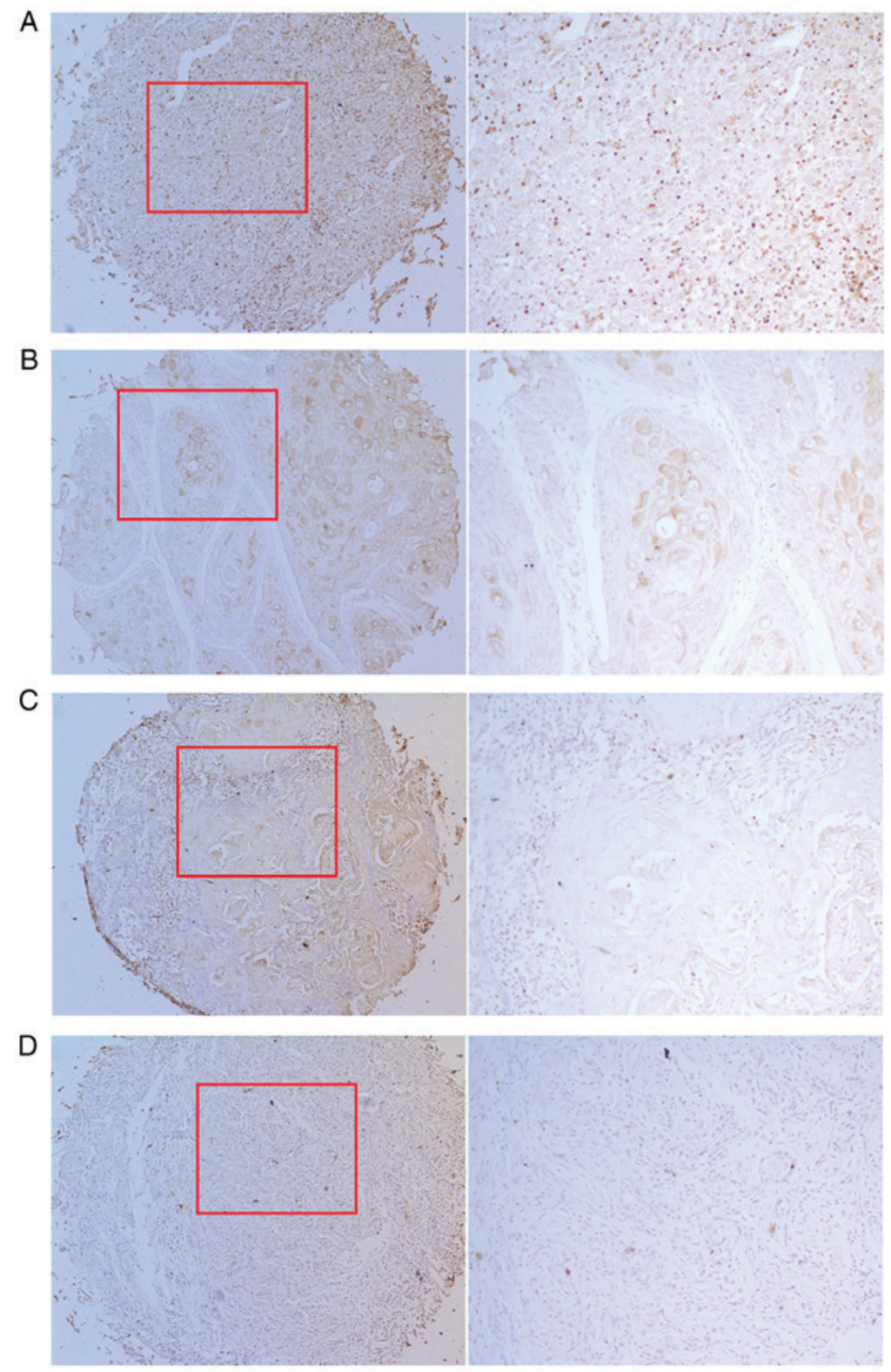

Figure 2. Representative images of nRUNX3 and sRUNX3 staining in tongue SCC tissues (left panel, magnification, x100; right panel, magnification, x200). (A) Positive nuclear staining of nRUNX3 and sRUNX3 in tongue SCC tissues. (B) Cytoplasmic nRUNX3 staining and negative sRUNX3 staining in tongue SCC tissues. (C) Negative nRUNX3 staining and positive sRUNX3 staining in tongue SCC tissues. (D) Negative nRUNX3 and sRUNX3 staining in tongue SCC tissues. nRUNX3, RUNX3 in carcinoma nests; RUNX3, runt-related transcription factor 3; SCC, squamous cell carcinoma; sRUNX3, RUNX3 in stroma. 
Table IV. Multivariate Cox regression analysis assessing the effects of covariates on overall survival in patients with tongue squamous cell carcinoma.

\begin{tabular}{llr}
\hline & \multicolumn{2}{c}{ (n=79 cases) } \\
\cline { 2 - 3 } Variable & HR $(95 \% \mathrm{CI})$ & P-value \\
\hline Age $(<60$ vs. $\geq 60$ years) & $1.57(0.76-3.23)$ & 0.219 \\
Sex (male vs. female) & $0.83(0.40-1.69)$ & 0.600 \\
Tumor size ( $\leq 2$ cm vs. $>2$ cm) & $1.84(0.83-4.07)$ & 0.132 \\
Lymph node metastasis (N0 vs. N1/N2/N3) & $3.91(1.16-13.10)$ & 0.027 \\
Histological grade (I vs. II/III) & $1.11(0.48-2.58)$ & 0.803 \\
Clinical stage (I vs. II/III/IV) & $0.22(0.06-0.82)$ & 0.023 \\
nRUNX3 expression (negative vs. positive) & $3.15(1.04-9.51)$ & 0.042 \\
\hline
\end{tabular}

CI, confidence interval; HR, hazard ratio; nRUNX3, runt-related transcription factor 3 in carcinoma nests.

phenotype, with a high rate of local lymph node metastasis and recurrence following comprehensive therapy, compared with other subtypes of oral cancer (2). However, the molecular mechanisms underlying these aggressive behaviors remain unclear and the prognosis of tongue SCC requires further evaluation.

The RUNX3 gene is located on chromosome 1p36, which has been suggested to harbor numerous tumor suppressor genes that are frequently deleted in various types of human cancer, including oral SCC (21). Evidence has suggested that RUNX3 is causally associated with carcinogenesis, and its inactivation contributes to tumor metastasis by enhancing cell migration and invasion in human cancer $(6,22)$. The RUNX3 gene may be inactivated by two mechanisms: i) Promoter hypermethylation, which leads to gene silencing and the absence of RUNX3 expression; and ii) protein mislocalization, which refers to the cytoplasmic retention of RUNX3 protein in a nonfunctional form, instead of normal nuclear localization. These two mechanisms may affect the progression of a number of types of human cancer $(9,12,19)$. Hypermethylation of the RUNX3 gene promoter is a common event in oral SCC, including tongue SCC (17,23-25), and is significantly associated with lymph node involvement and tumor stage in tongue SCC (23). Downregulation of RUNX3 protein expression and protein mislocalization are additionally correlated with histological grades in oral SCC (19). Furthermore, cytoplasmic mislocalization of the RUNX3 protein has been reported to be more frequent compared with the absence of RUNX3 protein expression in oral SCC (19); however, has not been fully investigated in tongue SCC. In tongue SCC tissue, RUNX3 has been reported to be hypomethylated, whereas its mRNA expression is increased (23). These findings suggested that the function of RUNX3 in tongue SCC may be distinct from other subtypes of oral SCC, although this remains to be fully elucidated. The present study detected the protein expression levels of RUNX3 protein in tongue SCC tissues and demonstrated that protein expression was reduced compared with in paired non-cancerous tissues. TMA results of samples obtained from patients with tongue SCC demonstrated that $51.9 \%$ of cases exhibited cytoplasmic staining of RUNX3, whereas only $17.7 \%$ cases exhibited negative staining. These findings suggested that RUNX3 inactivation in tongue SCC may be due to protein mislocalization, rather than absence of expression.

The exact mechanism underlying RUNX3 protein mislocalization has not been clearly elucidated; however, it has been confirmed that RUNX3 protein cannot elicit tumor suppressive activity when the protein is restricted to the cytoplasm (5). Transforming growth factor- $\beta$ (TGF- $\beta$ ) is a tumor-suppressing factor and an upstream molecule of RUNX3, which can initiate RUNX3 protein translocation from the cytoplasm into the nucleus (6). In gastric cancer, it has been reported that the cytoplasmic retention of RUNX3 increases tumorigenicity when TGF- $\beta$-induced nuclear translocation of RUNX3 is impaired (9). Therefore, in the present study, immunofluorescence and immunocytochemistry were performed to locate the distribution of RUNX3 in tongue SCC cell lines. In agreement with TMA results, SCC25 and Cal27 cells exhibited low RUNX3 protein expression, which was predominately localized to the cytoplasm. These results further suggested that protein mislocalization of RUNX3 may serve an important role in the carcinogenesis of tongue SCC. Notably, some tongue SCC cells exhibited nuclear staining, whereas the majority of cells exhibited cytoplasmic staining. These results indicated the complexity of RUNX3 function in tongue SCC, which requires further elucidation. In previous studies, the restoration of RUNX3 could potentially inhibit the malignant behaviors of human cancer $(9,13,14)$. Therefore, it may be feasible to select RUNX3 as an antitumor target.

Molecular abnormities caused by cancer metastasis occur not only in tumor nests; however, additionally in the tumor stroma. Cancer cells detach from the primary tumor, invade the basement membrane and extracellular matrix, and transport within the blood or lymphatic vessels in the surrounding tumor stroma, finally arriving in the target organ to form metastasis foci. Interactions between the tumor and surrounding tissue, which consists of stromal cells, blood vessels and immune cells, are vital to tumor growth and spread $(26,27)$. Expression of certain proteins or microRNAs in the tumor stroma may be candidates as molecular biomarkers of prognosis $(28,29)$. Therefore, in the present study, nRUNX3 and 

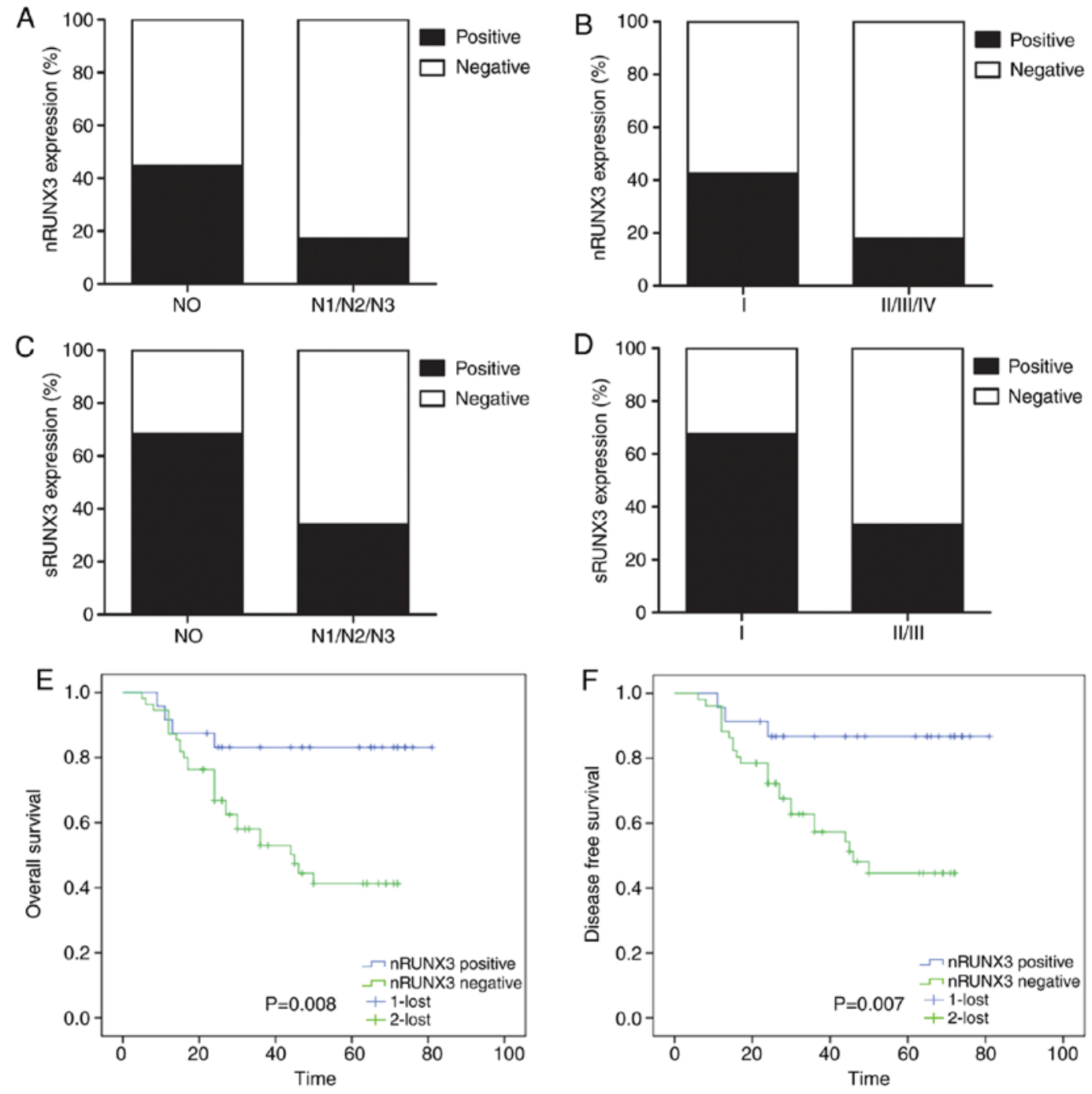

Figure 3. Negative expression of nRUNX3 is associated with poor survival rates of patients. (A) Reduced nRUNX3 expression was associated with lymph node metastasis $\left(\mathrm{P}=0.014 ; \chi^{2}\right.$ test). (B) Reduced nRUNX3 expression was associated with clinical stage $\left(\mathrm{P}=0.027 ; \chi^{2}\right.$ test). (C) Reduced sRUNX3 expression was associated with lymph node metastasis $\left(\mathrm{P}=0.003 ; \chi^{2}\right.$ test). (D) Reduced sRUNX3 expression was associated with clinical stage $\left(\mathrm{P}=0.003 ; \chi^{2}\right.$ test). (E) Negative nRUNX3 expression was associated with a poorer overall survival ( $\mathrm{P}=0.008$; log-rank test). (F) Negative nRUNX3 expression was associated with poorer disease-free survival ( $\mathrm{P}=0.007$; log-rank test). nRUNX3, RUNX3 in carcinoma nests; RUNX3, runt-related transcription factor 3; sRUNX3, RUNX3 in stroma.

SRUNX3 expression were distinctly evaluated; to the best of our knowledge, this has not been analyzed in previous studies. Inconsistent expression of RUNX3 was identified in tumor nests and stroma. Unlike nRUNX3, cytoplasmic staining was not identified in sRUNX3. Positive sRUNX3 nuclear staining was observed in $50.6 \%$ of cases, which was mostly distributed in the adjacent area between tumor nests and stroma. By analyzing their association with clinicopathological variables, it was revealed that nRUNX3 and sRUNX3 were significantly associated with lymph node metastasis. In addition, negative nRUNX3 expression, but not sRUNX3, worsened the overall and disease-free survival of patients. The univariate and multivariate Cox regression analyses confirmed that negative nRUNX3 was associated with poor prognosis, thus suggesting nRUNX3 may function as a prognostic biomarker for tongue SCC.

Numerous studies have reported that RUNX3 serves as an oncogene in head and neck SCC (HNSCC) $(30,31)$. However, functional mutation or protein mislocalization of RUNX3 has not been detected in HNSCC cells (31). In our previous study, it was identified that RUNX3 serves a tumor suppressor role by inhibiting cell migration, invasion and angiogenesis in oral SCC (18). As one subtype of oral SCC, the protein expression levels of RUNX3 were detected in tongue SCC tissues and cell lines. The results suggested that cytoplasmic mislocalization of the RUNX3 protein was a common event in tongue SCC. The findings of the present study additionally indicated that nRUNX3 expression may be a potential prognostic biomarker for tongue SCC. The limitations of the present study were the absence of adjacent normal tissues paired with tongue SCC tissues for the TMA analysis, and the limited number of fresh samples $(n=3)$ used to analyze the expression of RUNX3 in tumor and adjacent non-cancerous tissues.

\section{Acknowledgements}

The authors would like to thank Professor Laikui Liu from Jiangsu Key Laboratory of Oral Diseases, Affiliated Hospital of Stomatology, Nanjing Medical University for his professional suggestions for this article. 


\section{Funding}

The study was funded by the Priority Academic Program Development of Jiangsu Higher Education Institutions (grant no. PAPD2014-37) and The Jiangsu Provincial Commission of Health and Family Planning (grant no. Z201511).

\section{Availability of data and materials}

The datasets used and/or analyzed during the current study are available from the corresponding author on reasonable request.

\section{Authors' contributions}

W-NZ and YZ collected and analyzed the oral squamous cell carcinoma data of the patients. YD and W-NZ performed the histological examination of the tissue microarray, and the in vitro experiments. $\mathrm{WZ}$ and $\mathrm{YZ}$ evaluated the immunoreactions of the tissue microarray and analyzed the results. YW, XS and JB designed the study. W-NZ and YZ drafted the manuscript, and XS and JB revised the manuscript. All authors read and approved the final manuscript.

\section{Ethics approval and consent to participate}

The present study was performed under a protocol approved by the Institutional Review Boards of the Affiliated Hospital of Stomatology, Nanjing Medical University. All patients provided written informed consent prior to the study.

\section{Patient consent for publication}

Written informed consent for the publication of clinical data and accompanying images was obtained from the patient, patient's guardian or next of kin prior to the study.

\section{Competing interests}

The authors declare that they have no competing interests.

\section{References}

1. Scully C and Bagan J: Oral squamous cell carcinoma: Overview of current understanding of aetiopathogenesis and clinical implications. Oral Dis 15: 388-399, 2009.

2. Weatherspoon DJ, Chattopadhyay A, Boroumand S and Garcia I: Oral cavity and oropharyngeal cancer incidence trends and disparities in the United States: 2000-2010. Cancer Epidemiol 39. 497-504, 2015

3. Ng JH, Iyer NG, Tan MH and Edgren G: Changing epidemiology of oral squamous cell carcinoma of the tongue: A global study. Head Neck 39: 297-304, 2017.

4. Ghantous Y, Yaffi V and Abu-Elnaaj I: Oral cavity cancer: Epidemiology and early diagnosis. Refuat Hapeh Vehashinayim (1993) 32: 55-63, 71, 2015 (In Hebrew).

5. Hanai J, Chen LF, Kanno T, Ohtani-Fujita N, Kim WY, Guo WH, Imamura T, Ishidou Y, Fukuchi M, Shi MJ, et al: Interaction and functional cooperation of PEBP $2 / \mathrm{CBF}$ with Smads. Synergistic induction of the immunoglobulin germline Calpha promoter. J Biol Chem 274: 31577-31582, 1999.

6. Subramaniam MM, Chan JY, Yeoh KG, Quek T, Ito K and Salto-Tellez M: Molecular pathology of RUNX3 in human carcinogenesis. Biochim Biophys Acta 1796: 315-331, 2009.

7. Ito Y: Oncogenic potential of the RUNX gene family: 'Overview' Oncogene 23: 4198-4208, 2004.
8. Tonomoto Y, Tachibana M, Dhar DK, Onoda T, Hata K, Ohnuma H, Tanaka T and Nagasue N: Differential expression of RUNX genes in human esophageal squamous cell carcinoma: Downregulation of RUNX3 worsens patient prognosis. Oncology 73: 346-356, 2007.

9. Ito K, Liu Q, Salto-Tellez M, Yano T, Tada K, Ida H, Huang C, Shah N, Inoue M, Rajnakova A, et al: RUNX3, a novel tumor suppressor, is frequently inactivated in gastric cancer by protein mislocalization. Cancer Res 65: 7743-7750, 2005.

10. Soong R, Shah N, Peh BK, Chong PY, Ng SS, Zeps N, Joseph D, Salto-Tellez M, Iacopetta B and Ito Y: The expression of RUNX3 in colorectal cancer is associated with disease stage and patient outcome. Br J Cancer 100: 676-679, 2009.

11. Yu GP, Ji Y, Chen GQ, Huang B, Shen K, Wu S and Shen ZY: Application of RUNX3 gene promoter methylation in the diagnosis of non-small cell lung cancer. Oncol Lett 3: 159-162, 2012.

12. Lau QC, Raja E, Salto-Tellez M, Liu Q, Ito K, Inoue M, Putti TC, Loh M, Ko TK, Huang C, et al: RUNX3 is frequently inactivated by dual mechanisms of protein mislocalization and promoter hypermethylation in breast cancer. Cancer Res 66: 6512-6520, 2006.

13. Bai J, Yong HM, Chen FF, Song WB, Li C, Liu H and Zheng JN: RUNX3 is a prognostic marker and potential therapeutic target in human breast cancer. J Cancer Res Clin Oncol 139: 1813-1823, 2013.

14. Kim BR, Kang MH, Kim JL, Na YJ, Park SH, Lee SI, Kang S, Joung SY, Lee SY, Lee DH, et al: RUNX3 inhibits the metastasis and angiogenesis of colorectal cancer. Oncol Rep 36: 2601-2608, 2016.

15. Chen H, Wang Z, Wang S, Zhang Z and Shi S: Effect and mechanism of RUNX3 gene on biological characteristics of human esophageal squamous cell carcinoma (ESCC). Med Oncol 32: 357, 2015.

16. Mei PJ, Bai J, Liu H, Li C, Wu YP, Yu ZQ and Zheng JN: RUNX3 expression is lost in glioma and its restoration causes drastic suppression of tumor invasion and migration. J Cancer Res Clin Oncol 137: 1823-1830, 2011.

17. Adelstein D, Gillison ML, Pfister DG, Spencer S, Adkins D, Brizel DM, Burtness B, Busse PM, Caudell JJ, Cmelak AJ, et al: NCCN guidelines insights: Head and neck cancers, version 2.2017. J Natl Compr Canc Netw 15: 761-770, 2017.

18. Zhou WN, Du YF, Bai J, Song XM, Zheng Y, Yuan H, Zhang W, Zhang ZD and Wu YN: RUNX3 plays a tumor suppressor role by inhibiting cell migration, invasion and angiogenesis in oral squamous cell carcinoma. Oncol Rep 38: 2378-2386, 2017.

19. Gao F, Huang C, Lin M, Wang Z, Shen J, Zhang H, Jiang L and Chen Q: Frequent inactivation of RUNX3 by promoter hypermethylation and protein mislocalization in oral squamous cell carcinomas. J Cancer Res Clin Oncol 135: 739-747, 2009.

20. Myers JN, Elkins T, Roberts D and Byers RM: Squamous cell carcinoma of the tongue in young adults: Increasing incidence and factors that predict treatment outcomes. Otolaryngol Head Neck Surg 122: 44-51, 2000.

21. Lefeuvre M, Gunduz M, Nagatsuka H, Gunduz E, Al Sheikh Ali M, Beder L, Fukushima K, Yamanaka N, Shimizu K and Nagai N: Fine deletion analysis of 1p36 chromosomal region in oral squamous cell carcinomas. J Oral Pathol Med 38: 94-98, 2009.

22. Chen F, Liu X, Bai J, Pei D and Zheng J: The emerging role of RUNX3 in cancer metastasis (Review). Oncol Rep 35: 1227-1236, 2016.

23. Supic G, Kozomara R, Jovic N, Zeljic K and Magic Z: Hypermethylation of RUNX3 but not WIF1 gene and its association with stage and nodal status of tongue cancers. Oral Dis 17: 794-800, 2011.

24. de Freitas Cordeiro-Silva M, Stur E, Agostini LP, de Podestá JR, de Oliveira JC, Soares MS, Mendonça EF, Gouvea SA, Von Zeidler SV and Louro ID: Promoter hypermethylation in primary squamous cell carcinoma of the oral cavity and oropharynx: A study of a Brazilian cohort. Mol Biol Rep 39: 10111-10119, 2012.

25. Zhang S, Feng XL, Shi L, Gong CJ, He ZJ, Wu HJ and Ling TY: Genome-wide analysis of DNA methylation in tongue squamous cell carcinoma. Oncol Rep 29: 1819-1826, 2013.

26. Hynes RO: Metastatic potential: Generic predisposition of the primary tumor or rare, metastatic variants-or both? Cell 113: 821-823, 2003.

27. Lambert AW, Pattabiraman DR and Weinberg RA: Emerging biological principles of metastasis. Cell 168: 670-691, 2017. 
28. Hedbäck N, Jensen DH, Specht L, Fiehn AM, Therkildsen MH Friis-Hansen L, Dabelsteen E and von Buchwald C: MiR-21 expression in the tumor stroma of oral squamous cell carcinoma: An independent biomarker of disease free survival. PLoS One 9: e95193, 2014.

29. Shinriki S, Jono H, Ueda M, Obayashi K, Nakamura T, Ota K, Ota T, Sueyoshi T, Guo J, Hayashi M, et al: Stromal expression of neutrophil gelatinase-associated lipocalin correlates with poor differentiation and adverse prognosis in oral squamous cell carcinoma. Histopathology 64: 356-364, 2014.
30. Kudo Y, Tsunematsu T and Takata T: Oncogenic role of RUNX3 in head and neck cancer. J Cell Biochem 112: 387-393, 2011.

31. Tsunematsu T, Kudo Y, Iizuka S, Ogawa I, Fujita T, Kurihara H, Abiko Y and Takata T: RUNX3 has an oncogenic role in head and neck cancer. PLoS One 4: e5892, 2009.

(i)(9) This work is licensed under a Creative Commons cc) $\mathrm{EY}$ No ND Attribution-NonCommercial-NoDerivatives 4.0 International (CC BY-NC-ND 4.0) License. 